\title{
Modelling and Prediction of the Gross Mortality Rate in Ecuador
}

\author{
Mónica Mite ${ }^{1}$ t. Sandra Garcia-Bustos ${ }^{2}$, Marcela Pincay $^{3}$, Ana Debón ${ }^{4}$, Francisco Santoja ${ }^{5}$ \\ University of Guayaquil \\ Faculty of Mathematics and Physical Sciences, Guayaquil, Ecuador \\ ${ }^{2}$ Polytechnic University, ESPOL \\ ${ }^{2}$ Centro de Estudios e Investigaciones Estadísticas, Guayaquil, Ecuador \\ ${ }^{3}$ Universidad Estatal del Sur de Manabí \\ ${ }^{4}$ Universidad Politécnica de Valencia \\ Centro de Gestión de la Calidad y del Cambio, Valencia, España \\ ${ }^{5}$ Universidad de Valencia \\ Departamento de Estadística e Investigación Operativa, Valencia, España \\ ${ }^{{ }^{+}}$Corresponding author's email: monica.miteleon [AT] ug.edu.ec
}

\begin{abstract}
This paper presents the results obtained from the modelling of the mortality data in Ecuador from 1990 to 2010, using the StMoMo library in the open source programming language $R$. This library was developed based on the Generalized Age-Period-Cohort Models (GAPC), among which is the Lee-Carter model, which has been widely applied in the actuarial area. The gross mortality rate of men and women in an age range of 1 to 85 years was modelled for the data of Ecuador, in the period 1990-2010. Of a total of eight models, two models have been selected because they present a good fit of the data for both genders. The first is the basic model of Lee-Carter and the second, the Plat model, which incorporates the cohort effect. A comparison was made with the two models to determine which one has a better forecast in a horizon of 20 years for specific ages. Both models show and predict the decrease in mortality in Ecuador of both genders, a decrease that is more pronounced, in general, for women at certain ages. In determining the uncertainty of the models, the bootstrap technique was used to define the confidence intervals of the adjusted model. The GAPC and ARIMA models were also compared; the former improve the mortality forecasting.
\end{abstract}

Keywords---- Mortality, Lee-Carter Model, Plat Model, StMoMo, ARIMA

\section{INTRODUCTION}

One of the roles that a country has is to know its population dynamics to determine the structure of the population, and thus make appropriate decisions in the economic, political and healthcare aspects. Mortality is one of the elements that make up this population dynamic and its study is a top priority for any nation.

In developed countries there has been a growth of the elderly population in recent decades, and in Latin America this pattern is emerging due to current advances in medicine and health insurance plans, which have helped improve the quality of life of people and reduce mortality rates, according to the Economic Center for Latin America and the Caribbean [1]. In 1970, half of these countries were trapped in economic underdevelopment and had not gone through a demographic transition, life expectancy was 55 years and the fertility rate was of 6 children per woman. Countries with a transitional demographic model such as India, Egypt, Morocco, Ecuador, Peru, Paraguay and the Dominican Republic face a double burden of communicable diseases, malnutrition and the increase of non-communicable diseases, often in combination with emerging diseases, such as HIV/AIDS or resurgent diseases such as malaria[2].

Therefore, the adjustment of mortality rate models in countries such as Ecuador is of great interest to researchers, and in particular to actuaries, because they are used to calculate premiums and reserves. Some authors such as: [3], [4] and [5] have made predictions and estimates of the mortality of the Ecuadorian population". In the work of "ShagñayHuaraca (2014) the model developed by Lee-Carter [6] is used to study Ecuadorian mortality during the period from 1990 to 2011 and concludes that the Lee Carter model improves the results of the life expectancy forecast, presenting differences in the mortality tables carried out by SENPLADES (National Secretariat of Planning and Development of Ecuador)". 
In this work we have used the data of the number of deaths and population of vital statistics records of Ecuador during the period 1990-2010 and ages from 1 to 85+ years provided by the National Institute of Statistics and Census of Ecuador (INEC) in order to adjust the GAPC models to the Ecuadorian mortality rate. Ecuador is a Latin American country with little research in the stochastic modelling of mortality data, for this reason this work is relevant for demographers, actuaries and statisticians, because mortality modelling can be used to construct mortality tables that can be used in the public health system, public and private insurers, and in the economic and social development of Ecuador.

Modelling the data was done with the use of the StMoMo package developed by Villegas [7] in the R language [8]. This StMoMo package includes functions to adjust GAPC mortality models, measure their goodness of fit and make predictions.

The structure of this work is as follows: Section 1 presents the data and notation. Section 2 presents the theoretical framework of the GAPC stochastic models and the implementation of the models using the StMoMo package. Section 3 presents the analysis of Ecuador's data. Section 4 details the adjusted models and the resulting forecasts. And finally, Section 5 presents the conclusions and recommendations.

\section{DATA AND NOTATION}

The population and mortality data of Ecuador was obtained from the web http://www.ecuadorencifras.gob.ec/nd of the National Institute of Statistics and Census (INEC) in June 2015. Because the censuses in Ecuador are carried out every ten years, the mortality data taken from the 1990, 2001 and 2010 censuses have been considered. For the years between the censuses, a linear interpolation was estimated according to the methodology proposed by [9]. The ages considered are in the age range of 1 to $85+(85+$ indicates that they are ages greater than or equal to 85 years). The notation used in this article is:

- $\quad D_{x t}$ is the random variable of the number of deaths at age $\mathrm{x}$ in year $\mathrm{t}$.

- $d_{x t}$ is the number of deaths observed

- $E_{x t}^{C}$ is the central population exposed to the risk of dying at age $\mathrm{x}$ in year $\mathrm{t}$

- $E_{x t}^{0}$ is the initial population exposed to the risk of dying

- $\hat{q}_{x t}=\frac{d_{x t}}{E_{x t}^{0}}$ is the probability of death estimated at one year of the individual age $\mathrm{x}$ in year $\mathrm{t}$

- $\widehat{m}_{x t}=\frac{d_{x t}}{E_{x t}^{C}}$ is the gross central rate of estimated mortality at age $\mathrm{x}$ in year $\mathrm{t}$.

- $\mu_{x t}$ is the force of mortality in year $\mathrm{t}$ at any age $\mathrm{x}$. It is the instantaneous rate of death.

The force of mortality and the central mortality rate coincide under the hypothesis that the force of mortality is constant for age $x$ to $x+1$ to the calendar year from $t$ to $t+1$, and that the population is stationary. The probability of death can be obtained using equation 1 ,

$$
q_{x t}=1-\exp ^{-\mu_{x t}} \quad=1-\exp ^{-m_{x t}}
$$

The data of number of deaths and population exposed to the risk of dying form a matrix, where the rows are the ages $x=x_{1}, \ldots ., x_{k}$ and the columns are the years $t=t_{1}, \ldots . t_{n}$. When only $E_{x t}^{C}$ or $E_{x t}^{0}$ are available, we can use the following equation to complement,

$$
E_{x t}^{0} \approx E_{x t}^{c}+\frac{1}{2} d_{x t}
$$

In our case, the ages used are in the interval of $x=1, \ldots \ldots, 85$ and the years $t=1990, \ldots \ldots, 2010$. People older than 85 years were added to the group of people of 85 years old, because in some cases several ages did not present records of deaths or population. With the data of deaths and population by age and sex, the gross mortality rate was obtained, with which this article has been worked.

\section{GENERALIZED STOCHASTIC MODELS - AGE-PERIOD-COHORT - GAPC}

According to [10] (Hunt and Blake, 2015), the mortality models proposed in the existing literature about Generalized Age-Period-Cohort models (GAPC), can be expressed as generalized linear and non-linear models [11].

GAPC stochastic models are composed of four components:

A. Random component. The number of $D_{x t}$ is modeled assuming a Poisson distribution or Binomial distribution, that is:

$$
D_{x t} \sim P o\left(E_{x t}^{C}, \mu_{x t}\right) \quad o \quad D_{x t} \sim B i\left(E_{x t}^{0}, q_{x t}\right)
$$


Being,

$$
\begin{aligned}
& \mu_{x t}=E\left(\frac{D_{x t}}{E_{x t}^{c}}\right) \\
& q_{x t}=E\left(\frac{D_{x t}}{E_{x t}^{0}}\right)
\end{aligned}
$$

B. Systematic component. Represented by the predictor

Where:

$$
\eta_{x t}=\alpha_{x}+\sum_{i=1}^{N} \beta_{x}^{(i)} \kappa_{t}^{(i)}+\beta_{x}^{(0)} \gamma_{t-x}
$$

The term $\alpha_{x}$ represents the static age function, which captures the general form of mortality by age.

The bilinear term $\sum_{i=1}^{N} \beta_{x}^{(i)} \kappa_{t}^{(i)}$ represents the age-period effect, where $\beta_{x}^{(i)}$ describes the variation in mortality with respect to age $\mathrm{x}$, and $\kappa_{t}^{(i)}$ is the index or trend of mortality that depends on time.

The term $\gamma_{t-x}$ represents the effects due to the cohort and is a function of the year of birth. The term $\beta_{x}^{(0)}$ can be a pre-specified function of age.

In the GAPC models, the mortality rate and cohort index are considered stochastic processes.

C. Link function. It connects the random component to the systematic component. It is given by the equation,

$$
g\left(E\left(\frac{D_{x t}}{E_{x t}}\right)\right)=\eta_{x t}
$$

The canonical links are: log for the Poisson distribution, and logit for the Binomial distribution.

D. The set of restrictions of the parameters. For models to present a single solution, restrictions are included through a constraint function denoted by $v(\theta)$ which ensures the model's identifiability.

The StMoMo library uses the GNM library [12], for the estimation of the parameters according to the maximum likelihood method in [13].

Of the eight GAPC models (Lee-Carter, Renshaw-Haberman, Age-Period-Cohort, Cairns-Blake-Dowd, CairnsBlake-Dowd with cohort effect, Quadratic Cairns-Blake-Dowd with cohort effect, Decreasing with cohort-time effect and Plat), this paper will present only the two models that best fit the data of the gross mortality rate of Ecuador.

\subsection{Lee-Carter Model (LC)}

The structure of the model proposed by [6] presents the predictor given by equation 3 . Where $\alpha_{x}$ is the term that depends on the age of death, $\beta_{x}^{(1)}$ the term presents the mortality rate of change in time for each age $\mathrm{x}$, and $\kappa_{t}^{(1)}$ is the trend of the mortality over the years

$$
\eta_{x t}=\alpha_{x}+\beta_{x}^{(1)} \kappa_{t}^{(1)}
$$

This model uses the logarithmic function as a link function. To estimate the mortality trend $\left(\kappa_{t}^{(1)}\right)$ time series models can be used [14]. The values of $\kappa_{t}^{(1)}$ have been modeled according to the Box-Jenkins methodology [15], that is, with $\operatorname{ARIMA~(p,~d,~q)~models~of~time~series.~}$

The Lee-Carter model presents an identifiability problem, which means that there is no single solution for the parameters. For a solution of equation (3) it can be transformed using arbitrary real constants $c_{1}$ and $c_{2}$ different from zero, as follows:

$$
\left(\alpha_{x}, \beta_{x}^{(1)}, \kappa_{t}^{(1)}\right) \rightarrow\left(\alpha_{x}+c_{1} \beta_{x}^{(1)}, \frac{1}{c_{2}} \beta_{x}^{(1)}, c_{2}\left(\kappa_{t}^{(1)}-c_{1}\right)\right)
$$

To ensure the identifiability of the estimated parameters of the model, the following set of restrictions is applied:

$$
\sum_{x} \beta_{x}^{(1)}=1 \quad y \quad \sum_{t} \kappa_{t}^{(1)}=0
$$




\subsection{Plat Model}

This model was structured by Richard Plat [16]. The Plat model combines the Cairns-Blake-Dowd (CBD) and LeeCarter models to form a new model. The predictor has the structure of equation 4 :

$$
\eta_{x t}=\alpha_{x}+\kappa_{t}^{(1)}+(\bar{x}-x) \kappa_{t}^{(2)}+(\bar{x}-x)^{+} \kappa_{t}^{(3)}+\gamma_{t-x}
$$

Where $(\bar{x}-x)^{+}=\max (\bar{x}-x, 0), \bar{x}$ is the average age in the data; $\alpha_{x}$ depends on the age of death; $\kappa_{t}^{(1)}$ represents the changes in the mortality rate across time for all ages; $\kappa_{t}^{(2)}$ allows changes in the mortality rate between ages to reflect historical observations that improve mortality in some ages; $\kappa_{t}^{(3)}$ is introduced to capture the dynamics of the mortality rate in younger ages and $\gamma_{t-x}$ captures the cohort effect in the same way as the Age-Period-Cohort model, that is, the mortality rates fluctuate according to the birth year and it is independent of age [17].

Factors $\kappa_{t}^{(2)}$ and $\kappa_{t}^{(3)}$ allow the model to not present a non-trivial correlation structure across ages. The set of restrictions to ensure identifiability are:

$$
\begin{gathered}
\sum_{t} \kappa_{t}^{(1)}=0, \quad \sum_{t} \kappa_{t}^{(2)}=0, \sum_{t} \kappa_{t}^{(3)}=0, \\
\sum_{t_{1}-x_{k}}^{t_{n}-x_{1}} \gamma_{c}=0, \sum_{t_{1}-x_{k}}^{t_{n}-x_{1}}=0 \quad y \quad \sum_{t_{1}-x_{k}}^{t_{n}-x_{1}}-x_{1} c^{2} \gamma_{c}=0
\end{gathered}
$$

The first three restrictions ensure that the period index is centered in zero and the last three ensure fluctuations of the cohort effect around zero. As in the LC model, the link function is logarithmic.

\section{ANALYSIS AND RESULTS OF THE DATA OF ECUADOR}

\subsection{Adjustment of the LC and Plat models for men}

In this section, the estimated parameters of the two GAPC models for men and women have been graphically represented. It starts with the behaviour of mortality for men. Figure 1 shows the graphs of the adjusted parameters the Lee-Carter and Plat models for the male population.

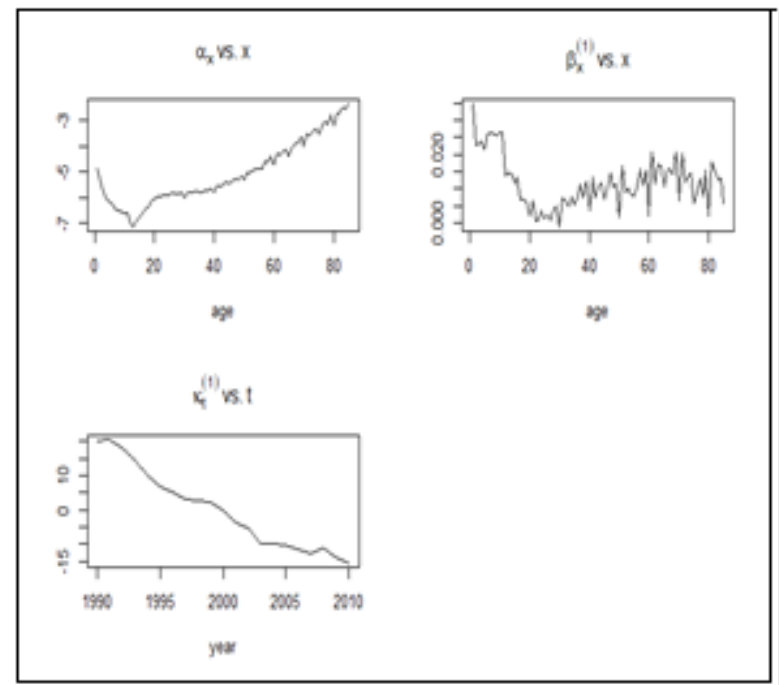

(a) the Lee Carter model for the male population

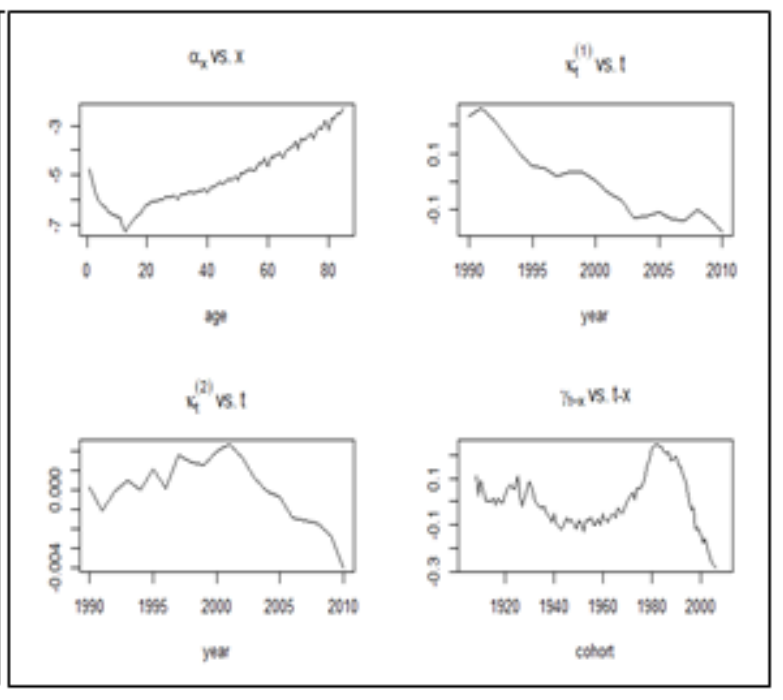

(b) The Plat model for the male population

Figure 1: Estimated parameters for the male population

The Figure 1 (a) shows the parameter $\alpha_{x}$ describes the behavior of the mortality rate through the ages of men. It is observed that the mortality rate at 1 year of age is high, then decreases rapidly until the age of 16 . At this point it grows 
again until the age of 20, keeping the curve slow and smooth until the age of 40 . Here it presents an over-mortality rate called "hump of accidents", which in the most developed countries occurs because of traffic accidents and that in Ecuador can have accidental causes and violence [3].

The general pattern of the parameter $\alpha_{x}$ in men is expected, a rapid decrease in the mortality rate for children (under 10 years of age) and increase during adulthood. Regarding the parameter $\beta_{x}$, the speed in the decrease in mortality in children and young people (under 20 years of age) can be observed. The positive values of this parameter show that mortality has decreased for all ages. The parameter $\kappa_{t}$ presents the trend of the mortality rate in the period studied which shows a pronounced decrease in the mortality rate until 2003 and then an even further decrease until 2010.

Figure 1 (b) shows the different parameters of the Plat model, in which the estimated parameter $\alpha_{x}$ indicates behavior of the mortality rate according to the age of the men. Its interpretation is similar to the LC model. The parameter $\kappa_{t}^{(1)}$ indicates that the mortality rate has a decreasing trend for all ages over the years. The parameter $\kappa_{t}^{(2)}$ shows variation of the mortality rate in the period analyzed indicating that the rate varies in different ages. The parameter $\gamma_{t-x}$ presents the cohort effect for the birth years during the 1990-2010 period.

\subsection{Adjustments of the LC and Plat model for women}

Figure 2 (a) presents the estimated parameters for the data of the women in the LC model. The parameter $\alpha_{x}$ indicates that the mortality rate decreases rapidly for children and young people, and then an increase is observed for adult ages. There is no excess mortality in women or "hump of accidents" as in the case of men. The parameter $\beta_{x}$ always takes positive values which indicates that the mortality rate decreases with age over time for all ages, and the parameter $\kappa_{t}$ shows that the trend of the mortality rate is decreasing in the period under review.

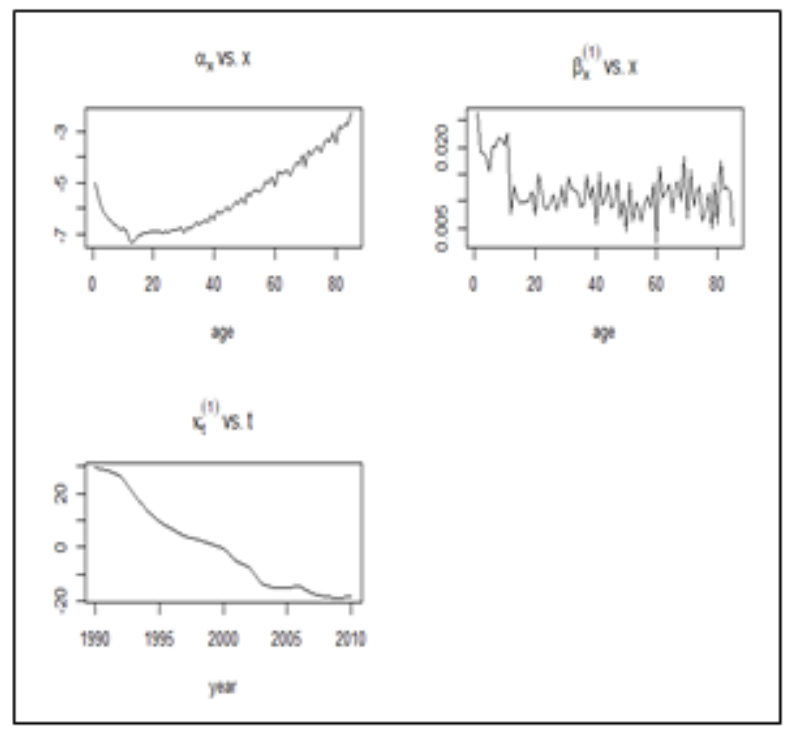

(a) The Lee Carter model for the female population

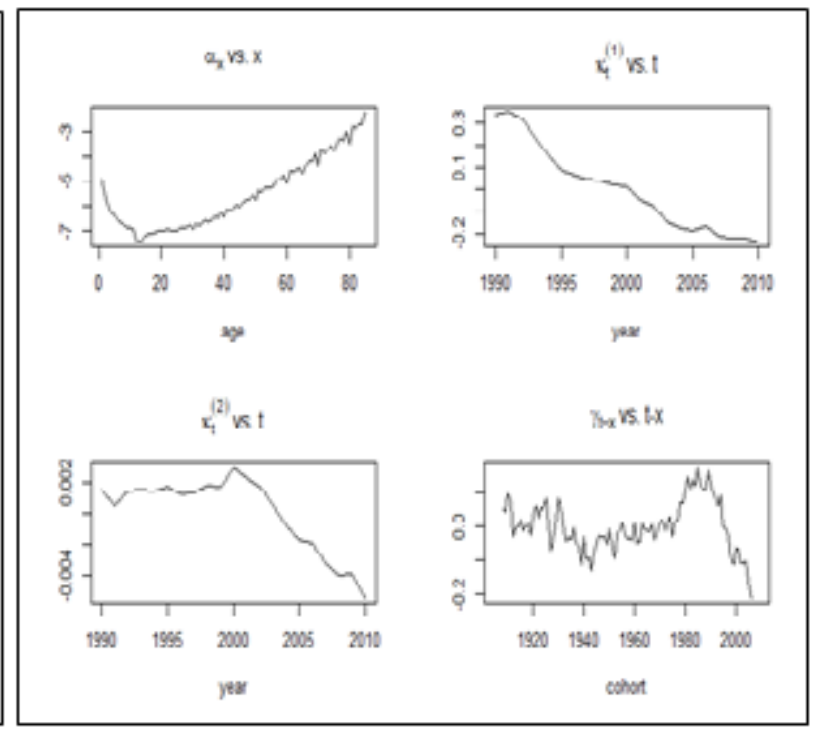

(b) The Plat model for the female population

Figure 2: Estimated parameters for the female population

The following figure 2 (b) shows the estimated parameters in the Plat model for the female population is similar to those of men. In this case the parameters decrease more sharply. The parameter $\alpha_{x}$ shows that the mortality rate at the age of 1 is high, then decreases until the person becomes approximately 16 years old, remaining constant from approximately 17 to 30 years of age, and then increasing as the age increases. The parameter $\kappa_{t}^{(1)}$ shows that the mortality rate has decreased in all ages in the time studied. The parameter $\kappa_{t}^{(2)}$ shows that the mortality rate in the period analyzed varies in different ages. The parameter $\gamma_{t-x}$ presents the cohort effect for the birth years during the 1990-2010 period.

In the graphical representation of the LC and Plat models, the estimated parameters of the models in both genders have similarities. In general, the trend of mortality rates is decreasing over the years, for both men and women. 


\subsection{Comparison of the models}

For the comparison of the LC and Plat stochastic models with the mortality data of men and women in Ecuador, the Bayesian Information Criterion (BIC) has been used, which is one of the most used for the selection of models (Akaike (1977) and Schwarz (1978)). The BIC value is a measure of goodness of fit that penalizes the number of parameters and avoids over-parameterization. This criterion is a function of the logarithm of the likelihood and the penalty term based on the number of model parameters.

$$
B I C=-2 \ln \ell(\phi)+\mathrm{K} \log N
$$

Since $\ell(\phi)$ is the estimated likelihood, $\mathrm{K}$ is the effective number of parameters and $\mathrm{N}$ is the number of observations. Table 1 shows the results of the statistics of adjustments of the two models with their respective number of degrees of freedom, the BIC (Bayes Information Criterion) and the execution time in seconds for the adjustment of the models for the male population in Ecuador.

According to the BIC criterion presented in Table 1, the two models show similar values, although there is a slight improvement in the fit obtained with the Plat model.

Table 1. Comparison of the models for the men population according to the BIC statistic

\begin{tabular}{|c|l|c|c|}
\hline Models & $\begin{array}{l}\text { Degrees of } \\
\text { Freedom }\end{array}$ & BIC & $\begin{array}{l}\text { Time } \\
(\mathrm{sec})\end{array}$ \\
\hline LC & 189 & 20067.47 & 14 \\
\hline Plat & 221 & 19624.80 & 8 \\
\hline
\end{tabular}

Table 2 shows the values of the adjustments of the GAPC models with their respective degrees of freedom, the BIC and the execution time in seconds for the adjustment of the models for the female population in Ecuador. In this case the Plat model presents the lowest BIC.

Table 2. Comparison of the models for the female population according to the BIC statistic

\begin{tabular}{|c|c|c|c|}
\hline Models & $\begin{array}{l}\text { Degrees of } \\
\text { Freedom }\end{array}$ & BIC & $\begin{array}{l}\text { Time } \\
(\mathrm{sec})\end{array}$ \\
\hline LC & 189 & 18913.89 & 15 \\
\hline Plat & 221 & 18205.19 & 8 \\
\hline
\end{tabular}

\subsection{Analysis of Results}

To select the model that best fits the mortality data in Ecuador, a graphic analysis of deviance residuals was performed. These residuals are defined according to the Poisson or Binomial random component by equations 6 and 7 respectively.

$$
\begin{aligned}
& \operatorname{dev}(x, t)=2 d_{x t} \log \left(\frac{d_{x t}}{\hat{d}_{x t}}\right)-\left(d_{x t}-\hat{d}_{x t}\right) \\
& \operatorname{dev}(x, t)=2 d_{x t} \log \left(\frac{d_{x t}}{\hat{d}_{x t}}\right)+\left(E_{x t}^{0}-d_{x t}\right) \log \left(\frac{E_{x t}^{0}-d_{x t}}{E_{x t}^{0}-\hat{d}_{x t}}\right)
\end{aligned}
$$

The StMoMo library uses the residual function to obtain these residuals according to age, calendar year and year of birth. Figures 3 and 4 show the residuals of the two selected models. For the representation of the waste, the Heat-plot graphs have been considered. Figure 3 presents deviance residuals for the male population. The values of the residuals are distributed approximately randomly for different ages and years, which show the good behavior of the models, especially the Plat model. 


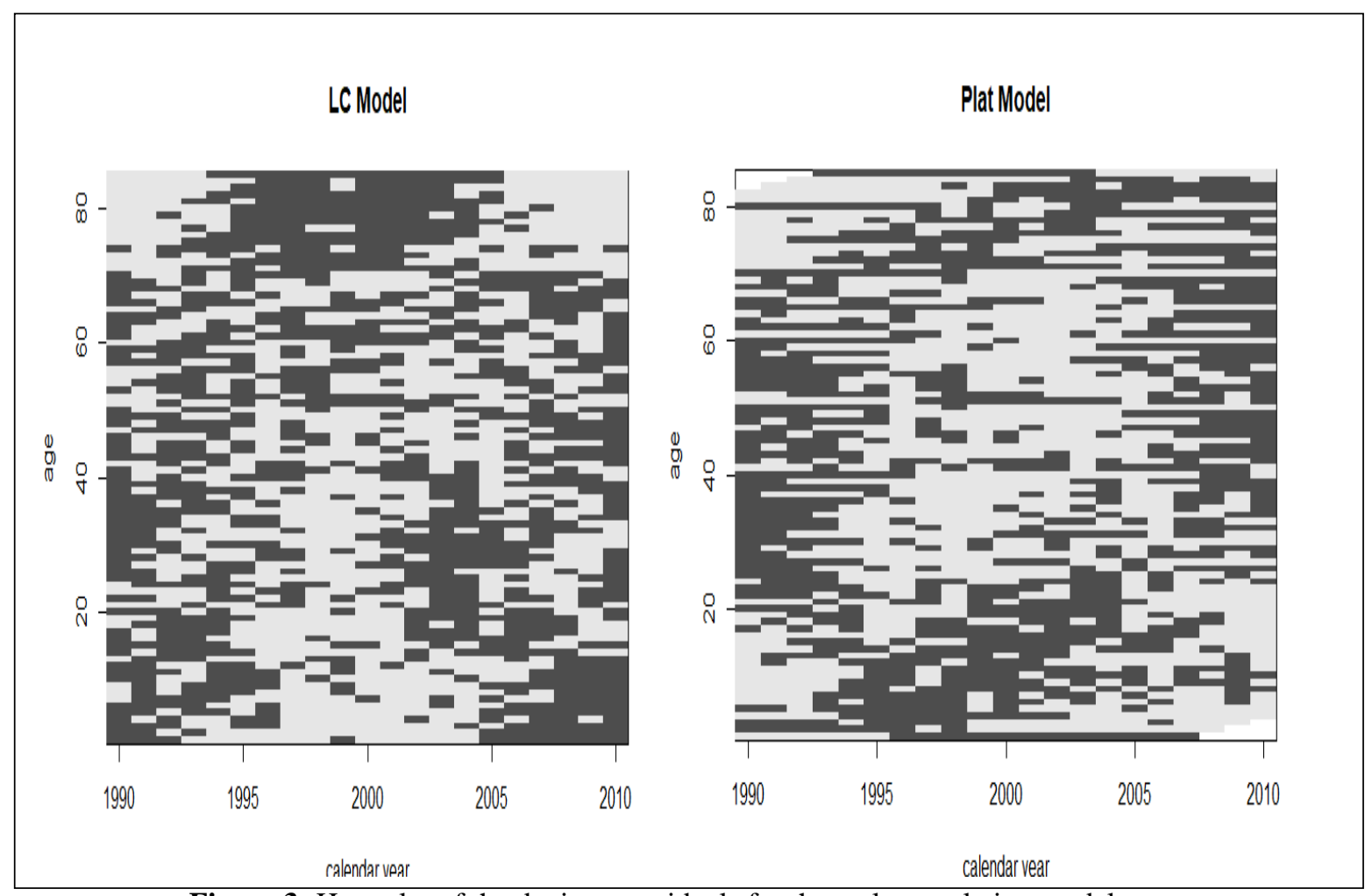

Figure 3: Heat-plot of the deviance residuals for the male population model

The deviance residuals corresponding to the modeling of the mortality rate for the female population are presented in Figure 4. In both models, randomness is observed, also, deviance residuals for men and women take values in the range of -3 to 3 . Even Though both models present similar results, the Lee-Carter model presents a simpler structure, while the Plat model has a more complex structure due to the cohort effect.

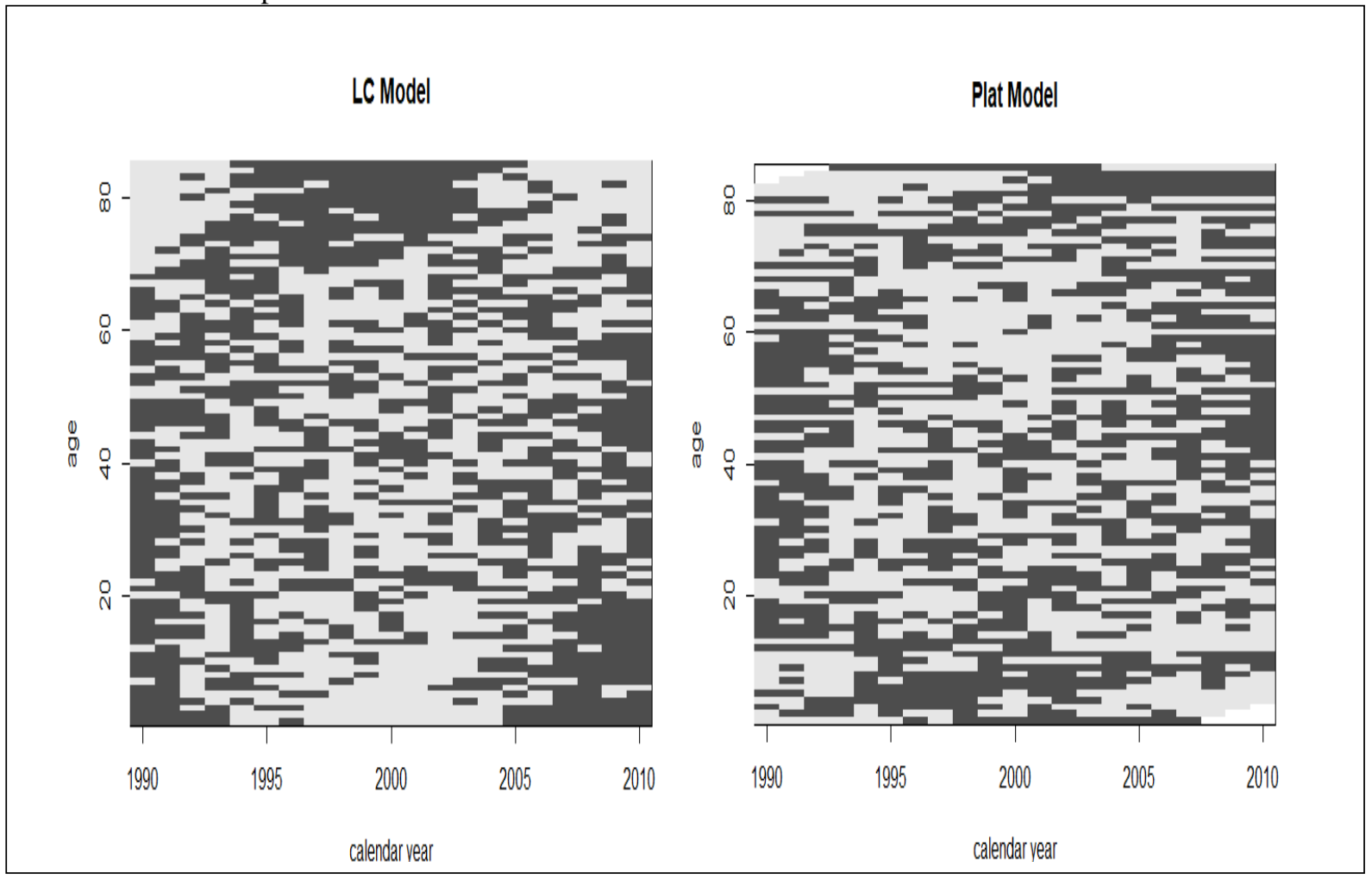

Figure 4: Heat-plot of the deviance residuals for the female population model 


\section{FORECAST OF THE LC AND PLAT STOCHASTIC MODELS}

In order to know the future behavior of the gross mortality rate of Ecuador and, considering that the modeling of the mortality rate through the GAPC models consider estimation of parameters that depend on time, such as the trend index $\kappa_{t}$, or the cohort effect index $\gamma_{t-x}$, the Box-Jenkins methodology will be used to forecast them. In this paper, the forecast with a time horizon of 20 years for the two selected models is presented. Figure 5 presents the predictions of mortality trends for the Ecuadorian data of men and women with a time horizon of 20 years for the LC model, highlighting that the mortality trend decreases with the years for men and women. In addition, the $95 \%$ prediction interval is not very wide.

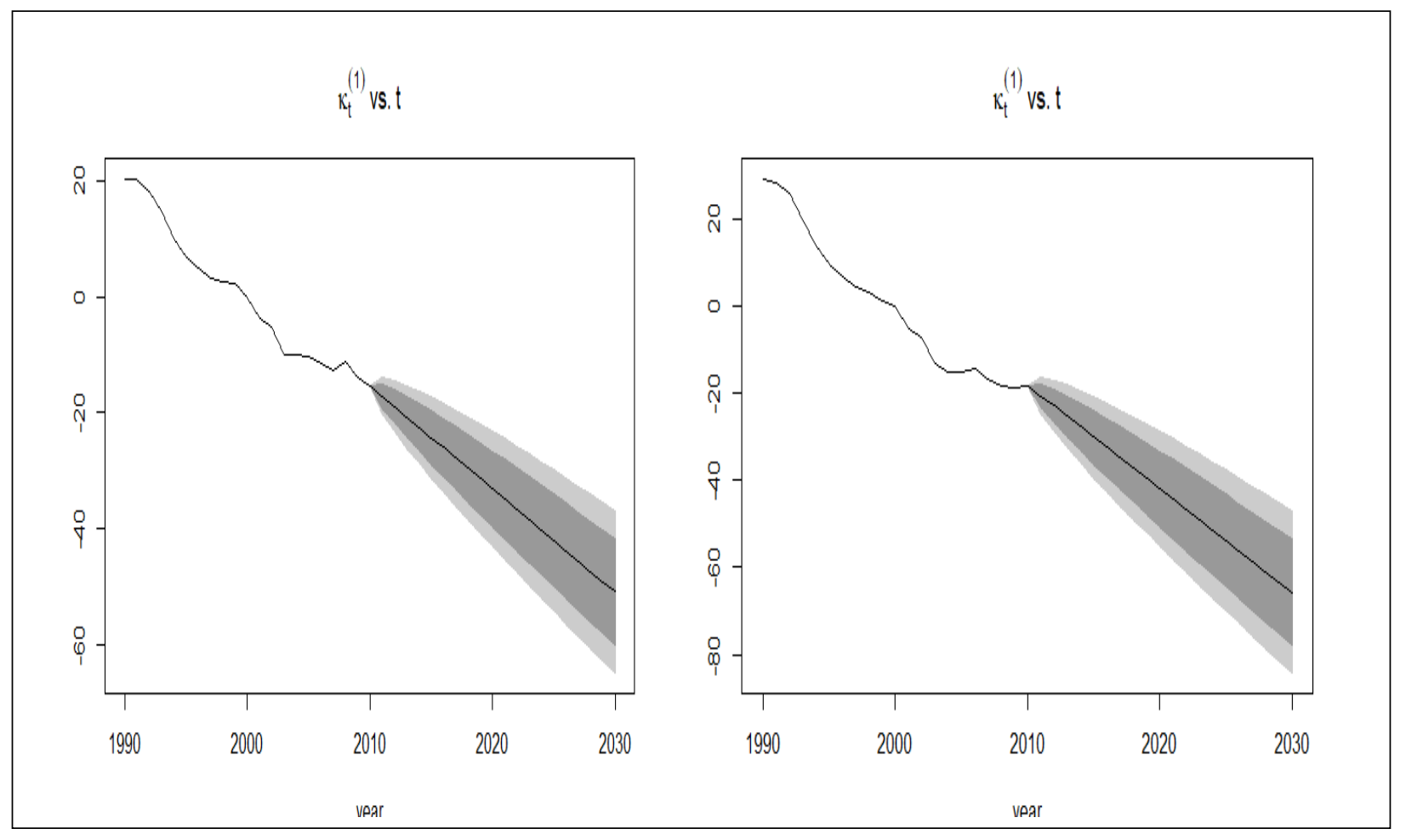

Figure 5: Lee-Carter Model Forecast

Figure 6 represents the forecast for men and women using the Plat model. It is observed that the trend of mortality falls rapidly and abruptly for the parameter $\kappa_{t}^{(1)}$, with a narrow prediction interval of $95 \%$. The forecast for the trend of the parameter $\kappa_{t}^{(2)}$ also decreases and the $95 \%$ prediction interval is wide. Consequently, the tendency of mortality in Ecuador tends to decrease in the time horizon of 20 years for both models. 


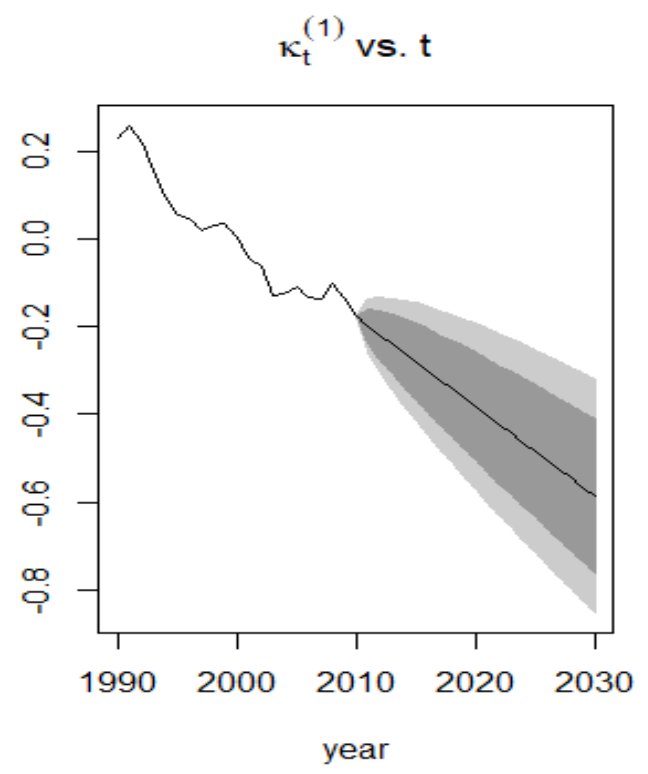

(a) Men

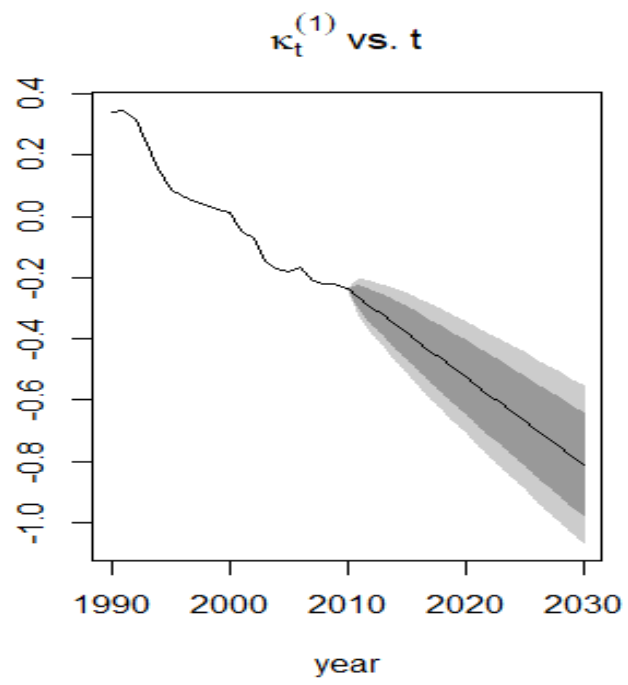

(b) Women
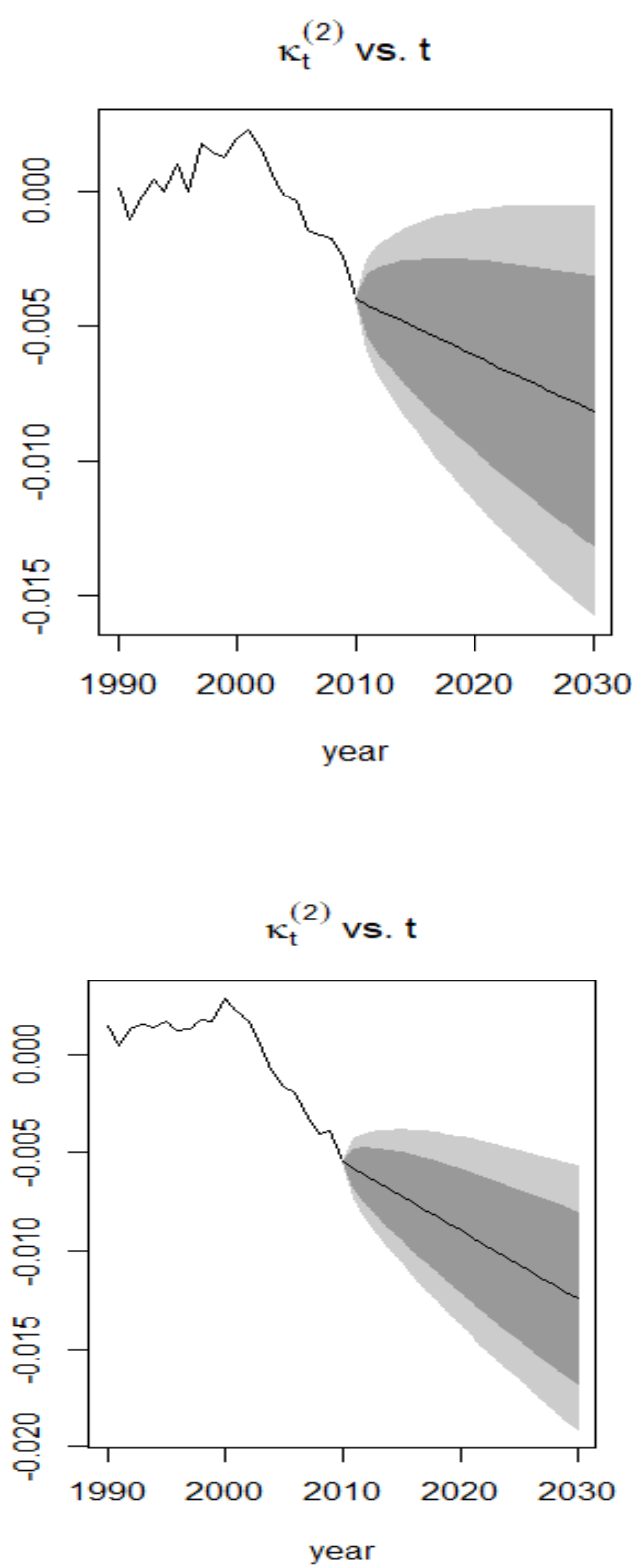

Figure 6: Plat Model Forecast 
Figure 7 shows the dynamics of the cohort effect for men and women of the mortality data in Ecuador. The forecast indicates a decrease in mortality due to the cohort effect.

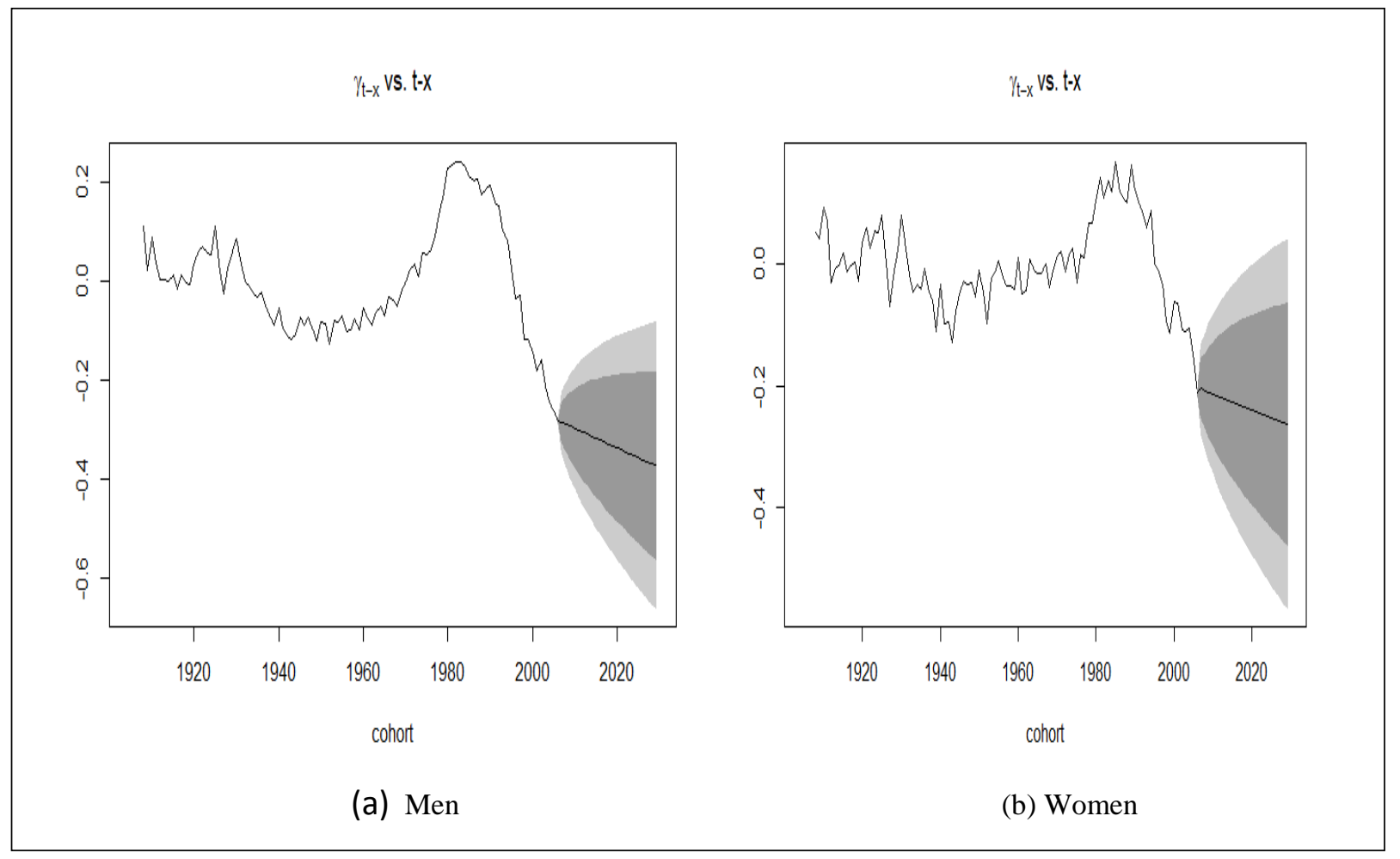

Figure 7: Forecast with the cohort effect of the Plat Model

\subsection{Uncertainty of the parameters of the mortality and forecast models}

Most stochastic mortality models have complex structures for the estimation of parameters. To quantify the different sources of uncertainty from the estimation of the parameters, bootstrap procedures are considered [18]. The bootstrap method was proposed by [19], and consists of obtaining samples from an original sample and estimating the parameters of the stochastic GAPC models for each of these samples with the purpose of constructing confidence intervals in the estimation of the parameters. Two bootstrap procedures are used, the parametric one proposed by and the nonparametric bootstrap by [20], who introduced them for the Lee-Carter model.

The StMoMo library implements the generic bootstrap function that executes the two types of procedures. In this work, 2000 bootstrap samples were used for each of the Lee-Carter and Plat models. The uncertainty was determined for three specific ages. 


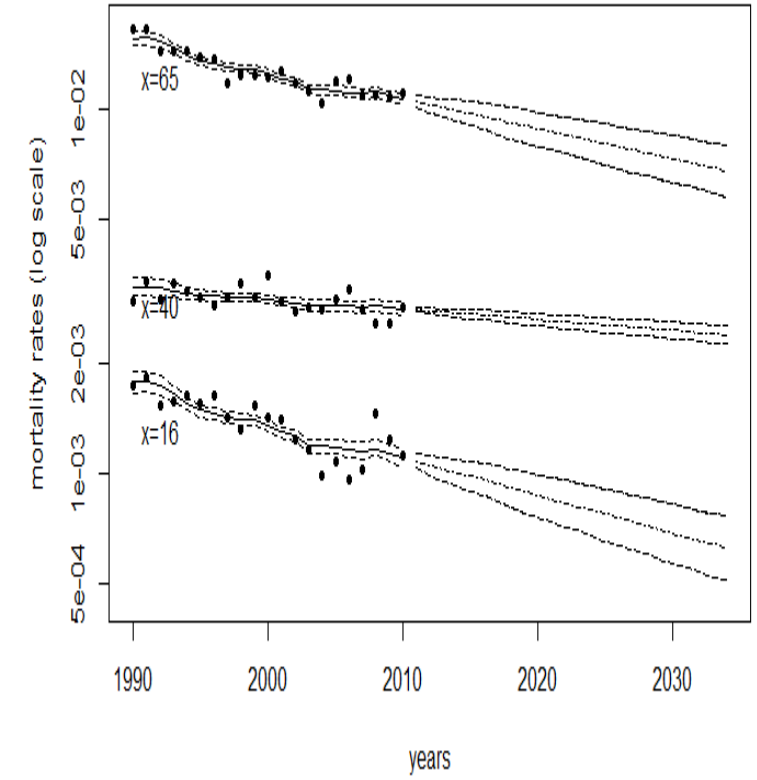

(a) Men

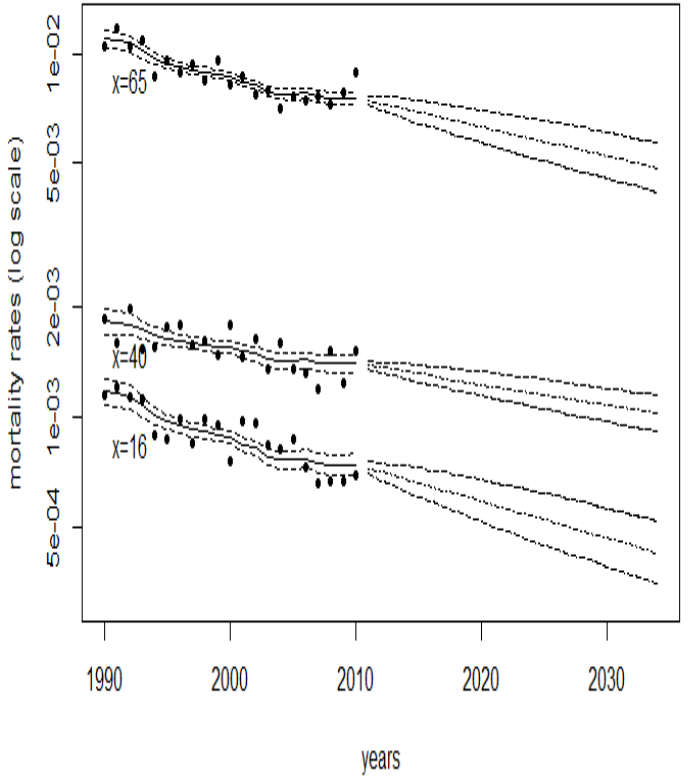

(b) Women

Figure 8: Mortality rates of ages 16, 40 and 65 of the LC Model with 95\% Confidence and forecast intervals

Figure 8 presents the forecasts for ages 16, 40 and 65 of the trend of mortality for the Lee-Carter model of men and women over a 20-year horizon. It is observed that for the age of 40, the mortality trend decreases very slowly in both men and women, and for the ages of 16 and 65 the mortality trend decreases with greater inclination in men and women. In this figure, the black points represent the observed gross mortality rates and, the red and black dashed lines are the adjusted rates that are prolonged to those predicted from the year 2010 with their respective red bandwidth that represents the $95 \%$ prediction interval. In this case, the width of the band is narrow.

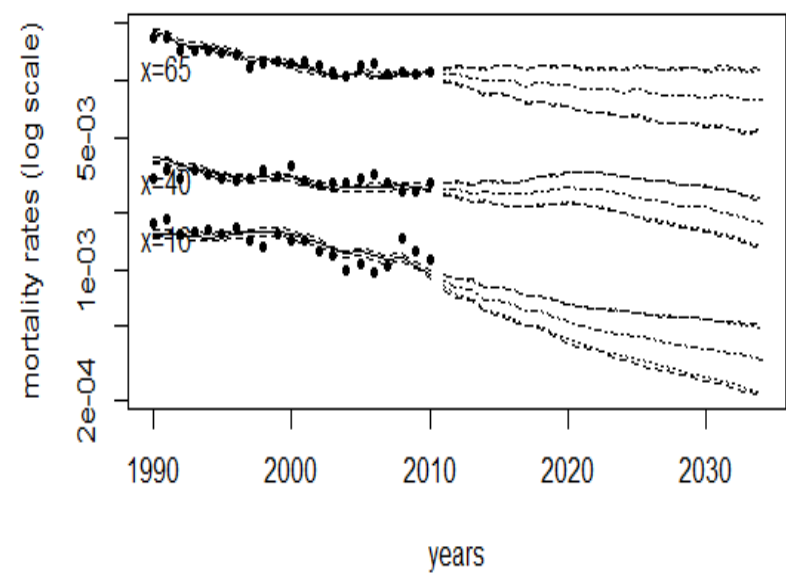

(a) Men

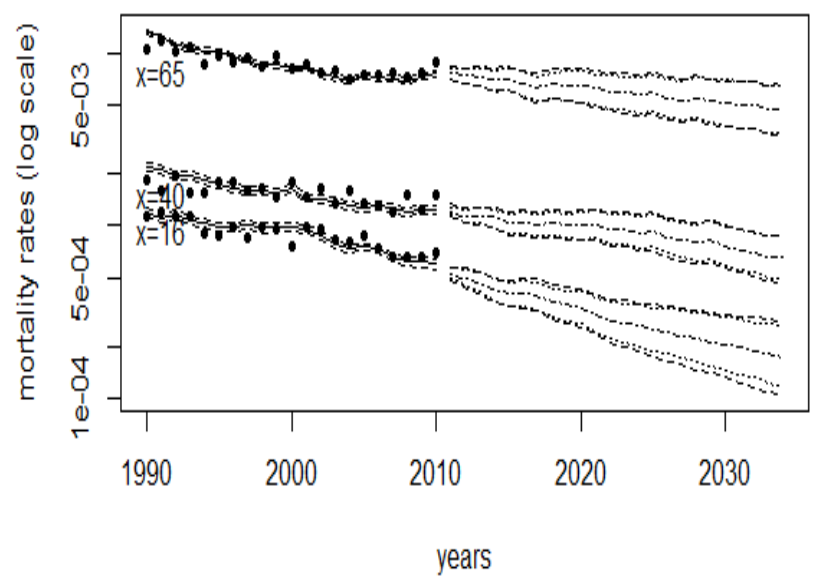

(b) Women

Figure 9: Mortality rates of ages 16, 40 and 65 of the Plat Model with 95\% Confidence and forecast intervals 
Figure 9 presents the forecast for the Plat model for men and women of specific ages of 16, 40 and 65 years of age. The black points represent the observed gross mortality rate, the dashed black lines represent the rate estimate and the prediction, and the red lines represent the confidence and prediction intervals of $95 \%$. The forecast of the mortality trend for the ages of 40 and 65 years decreases smoothly, and by the age of 16 years decreases rapidly. The bandwidth of the prediction interval is slightly more open with respect to the LC model, inferring that the uncertainty with the Plat model is greater than the LC model.

Additionally, we forecasted the trend of age-specific mortality rates for men and women using univariate time series. With the functions autoarima and forecast of the R forecast library [21], the ARIMA models were identified, then the proposed models were validated with the tests of normality, incorrectness and constant variance on the residuals.

With the resulting ARIMA models, the prediction was determined with a time horizon of 20 years. Figure 10 presents the observed rates, adjusted rates and the respective prediction intervals at $85 \%$ and $95 \%$, for the specific ages of 16,40 and 65 years for men and women.

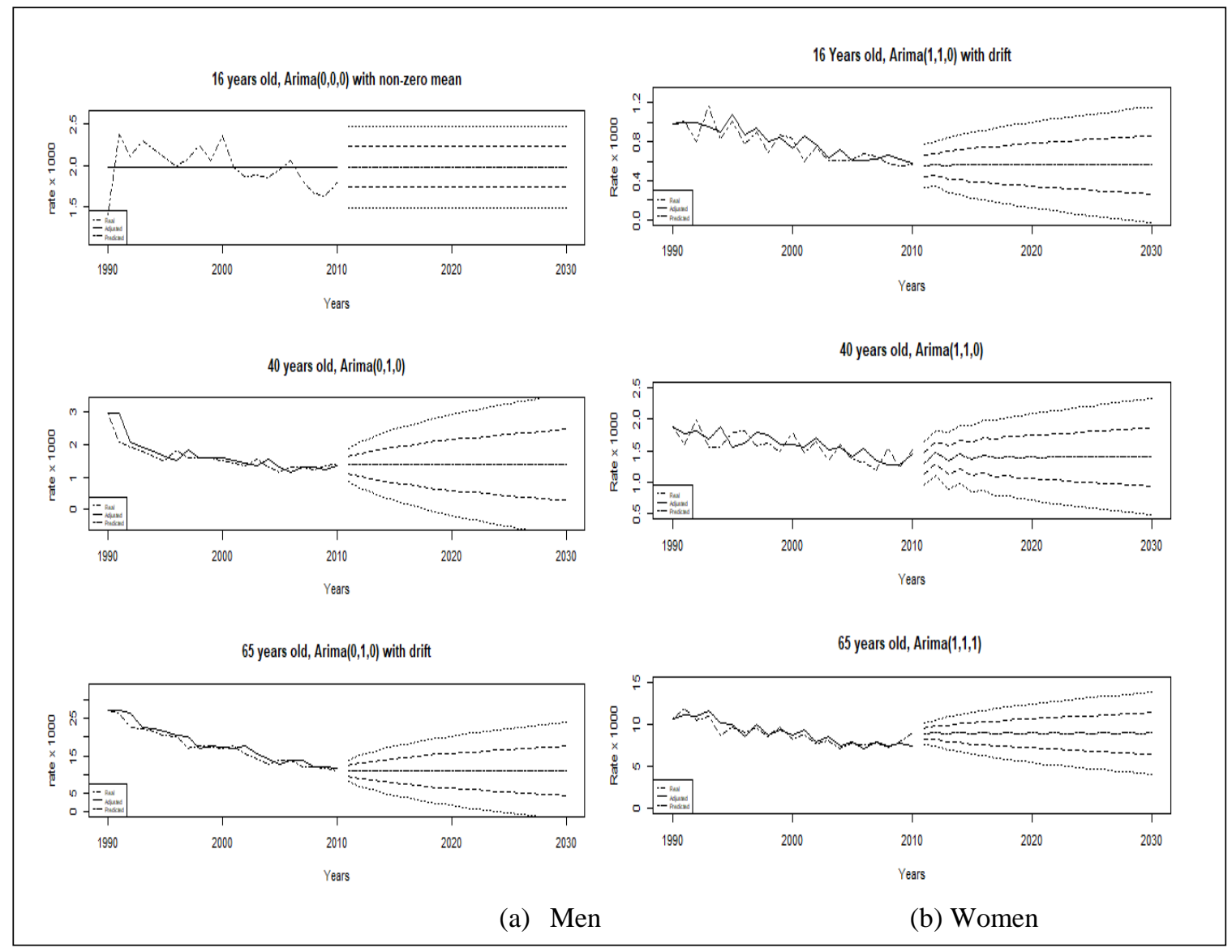

Figure 10: Forecasts of the gross mortality rate using the ARIMA models

In this figure, it is observed that the forecasts with the different ARIMA models depending on the specific age, the mortality rate remains constant in the projected time horizon. Table 3 shows the goodness-of-fit statistics of ARIMA models for specific ages, such as: Mean Error (ME), Root Mean Square Error (RMSE) and Mean Absolute Percentage Error (MAPE). Forecast errors are low values in ARIMA models of specific ages for men and women. These ages were considered to compare this methodology with the LC and Plat models.

Table 3. Measures of goodness of fit of the ARIMA Models

\begin{tabular}{|c|c|c|c|c|c|c|}
\hline & \multicolumn{3}{|c|}{ Men } & \multicolumn{3}{c|}{ Women } \\
\hline Age (years) & EM & RMSE & MAPE & EM & RMSE & MAPE \\
\hline 16 & 0.00 & 0.243 & 10.28 & 0.03 & 0.104 & 10.58 \\
\hline 40 & 0.07 & 0.239 & 9.31 & 0.03 & 0.163 & 9.21 \\
\hline 65 & 0.77 & 1.437 & 6.18 & 0.10 & 0.587 & 4.96 \\
\hline
\end{tabular}




\section{CONCLUSIONS AND DISCUSSION}

This paper presents the application of the StMoMo library with mortality data in Ecuador for men and women. The modeling of the data was done using the Lee-Carter and Plat models. These models were compared with the ARIMA models for three specific ages. Of the eight models mentioned at the beginning, only the two models that have presented a better fit to the Ecuadorian mortality rate have been analyzed: The Lee-Carter model presents a simple structure and its parameters are easily to interpret, and the Plat model that has a more complex structure but captures the cohort effect.

The modeling of the gross mortality rate with the Lee-Carter and Plat models shows that the trend has decreased for men and women over time, with the mortality trend being lower in women than in men. Regarding age, it is identified that mortality increases exponentially after 40 years and there is a greater risk in men than in women because they present the so-called "hump of accidents".

In order to measure the uncertainty in the estimation of the parameters of the Lee-Carter and Plat models, the confidence intervals of the estimation of parameters were determined to construct prediction and forecast intervals for the mortality rates of Ecuador that is presented in Figures 8 and 9 where it is observed that these intervals coincide. The following table 4 presents the mortality rates in 2010 for men and women of the three specific ages, as well as the predicted rates over the 20 -year horizon of the two models.

Table 4. Comparison observed rate and forecasted rate

\begin{tabular}{|c|c|c|c|c|c|c|}
\hline \multirow{2}{*}{$\begin{array}{c}\text { Ages } \\
\text { (years) }\end{array}$} & \multirow{2}{*}{ Observed rate 2010 (\%) } & \multicolumn{3}{|c|}{ Forecasted rate 2030 (\%) } \\
\cline { 2 - 7 } & Men & Women & Men & Women & Men & Women \\
\hline 16 & 0.112 & 0.069 & 0.062 & 0.038 & 0.047 & 0.021 \\
\hline 40 & 0.280 & 0.152 & 0.236 & 0.106 & 0.259 & 0.075 \\
\hline 65 & 1.105 & 0.891 & 0.745 & 0.611 & 0.916 & 0.623 \\
\hline
\end{tabular}

The decrease in mortality rates forecasted for the year 2030 for the ages of 16 and 65 years is considerable in men and women, unlike the age of 40 years where the rate decreases slowly in men and almost in half in women according to the Plat model. Both models show that the mortality rate tends to decrease in the coming years.

The comparison between the Lee-Carter and Plat stochastic models with the ARIMA models reveals that the GAPC models present better fit and forecasts than the ARIMA models in the specific ages according to the measures of goodness of fit. Although the prediction errors presented in Table 3 are small, the prediction of the mortality trend for the specific ages analyzed remains constant over the time horizon as seen in Figure 10, so the Lee-Carter and Plat presents a better prediction of specific ages and the Box-Jenkins methodology does not make a good prediction.

In general, in the modeling of mortality in Ecuador, it is concluded that the trend of the gross mortality rate has decreased in the period studied and is expected to decrease in the next 20 years for men and women. This decrease is due to the advances in medicine, the new strategies considered in the public and private health system, and the different construction environments that are related to providing the best hygienic and health conditions of the population in the country. It should be noted that this work is the first to be carried out applying the two stochastic models of Lee-Carter and Plat, as well as the work done by Shagñay-Huaraca (2014) which used the Lee-Carter model for the mortality data of Ecuador and by which we consider that these conclusions are important for the demographic and actuarial sectors.

In order to improve the prediction accuracy of the mortality rate in Ecuador in future research, new models should be developed considering age, sex, province, economic factor, etc. and thus determine the demographic behaviour of the population.

\section{REFERENCES}

[1] "ECLAC (2015). Economic Center for Latin America and the Caribbean 2015, URL https://www.cepal.org/es/publicaciones/39965-panorama-social-america-latina- 2015," p. 39965, 2015.

[2] B. K. Defo, "Demographic, epidemiological, and health transitions: are they relevant to population health patterns in \{Africa\}?," Glob. Health Action, vol. 7, 2014.

[3] P. D. Shagñay-Huaraca, "Ajuste y estimación de tablas de mortalidad dinámicas de la población ecuatoriana hasta el año 2030," Quito: EPN, 2014., 2014.

[4] H. C. and K. Lara, "CONSTRUCCIÓN DE TABLAS DE MORTALIDAD DE LA POBLACIÓN 
ECUATORIANA CON BASE EN EL CENSO 2001 Y ESTADÍSTICAS VITALES,” Consult. CIMACYT, pp. $1-7,2004$.

[5] J. Sanchez, "Construcción de una tabla de mortalidad para la población ecuatoriana," Repos. Dsp., 2000.

[6] R. D. Lee and L. Carter, "Modelling and forecasting \{U.S.\} mortality," J. Am. Stat. Assoc., vol. 87, no. 419, pp. 659-671, 1992.

[7] A. M. Villegas, P. Millossovich, and V. K. Kaishev, "StMoMo: An R Package for Stochastic Mortality Modelling." 2016.

[8] R Core Team, "R: A Language and Environment for Statistical Computing.” Vienna, Austria, 2015.

[9] A. Delwarde and M. Denuit, "Importance de la période d'observation et des ages consideres dans la projection de la mortalité selon la methode de Lee-Carter.," Belgian Actuar. Bull., vol. 1, no. 1, pp. 1-21, 2003.

[10] A. Hunt and D. Blake, "On the structure and classification of mortality models," Pension Inst. Work. Pap., 2015.

[11] I. D. Currie, "On fitting generalized linear and non-linear models of mortality," Scand. Actuar. J., vol. 2016, no. 4, pp. 356-383, 2016.

[12] H. Turner and D. Firth, "Generalized nonlinear models in R: An overview of the gnm package." 2015.

[13] N. Brouhns, M. Denuit, and J. K. Vermunt, "A \{Poisson\} log-bilinear regression approach to the construction of projected lifetables," Insur. Math. Econ., vol. 31, no. 3, pp. 373-393, 2002.

[14] A. Debón, F. Montes, and F. Martínez-Ruiz, "A geostatistical approach for dynamic life table: The effect of mortality on remaining lifetime and annuities.," Insur. Math. Econ., vol. 47, no. 3, pp. 327-336, 2010.

[15] G. E. P. and J. Box, Time Series Analysis: Forecasting and Control., Revised ed. 1970.

[16] R. Plat, "On stochastic mortality modeling," Insur. Math. Econ., vol. 45, no. 3, pp. 393-404, 2009.

[17] A. J. G. Cairns et al., "A quantitative comparison of stochastic mortality models using data from England and Wales and the United States," North Am. Actuar. J., vol. 13, no. 1, pp. 1-35, 2009.

[18] A. Debón, F. Montes, J. Mateu, E. Porcu, and M. Bevilacqua, "Modelling residuals dependence in Dymanic Life Tables," Comput. Stat. Data Anal., vol. 52, no. 3, pp. 3128-3147, 2008.

[19] B. Efron and R. J. Tibshirani, An introduction to the boostrap. Chapman \& Hall, New York \& London, 1993.

[20] M. Koissi, A. Shapiro, and G. Hognas, "Evaluating and extending the \{Lee-Carter $\}$ model for mortality forecasting confidence interval," Insur. Math. Econ., vol. 38, no. 1, pp. 1-20, 2006.

[21] R. Hyndman, "demography: Forecasting mortality and fertility data." 2005. 